licht, die im Verbund nicht anfallen würden. Auch andere, in diesem Beitrag nicht diskutierte Vorschläge hätten nicht dieselben strukturerhaltend korrigierenden Wirkungen: Das häufig zitierte Grabenwahlsystem ohne Verrechnung von Direkt- und Listenmandaten würde, wie auch das einfache Mehrheitswahlsystem, zu Wahlergebnissen führen, die weitab von dem liegen, was Wähler und Parteien in Deutschland gewohnt sind. Ein reines Verhältniswahlsystem mit Fünfprozentklausel würde die Proportionalität des Wahlergebnisses nicht wesentlich verbessern, und selbst wenn man offene Listen zuließe ${ }^{46}$, wäre dies auf Landesebene nur ein schwacher Ersatz für die jetzige Personenwahl in Einer-Wahlkreisen.

Die einzigartige Struktur des deutschen Wahlsystems hat sich über einen langen Zeitraum bewährt: Das Mischwahlsystem ermöglichte stabile, zumindest 1998 sogar alternierende Regierungsmehrheiten bei gleichzeitiger Wahrung von Parteien- und Landesproporz; es sorgte einerseits für die Entstehung geschlossener Parteiverbände mit nationaler Ausrichtung, ohne jedoch andererseits auf volksnahe, direkt gewählte Abgeordnete zu verzichten. Die unterschiedlichen Auswirkungen der Stimmabgabe in beiden Wahlgängen und die Gelegenheit zur individuellen Stimmgebung bieten dem Wähler Möglichkeiten, wie sie kaum ein anderes Wahlsystem bereithält. Seit Ende der achtziger Jahre hat sich das Mischwahlsystem deutscher Prägung zu einem wahren Exportschlager entwickelt. Es ist das meistadoptierte System der jüngeren Vergangenheit, und es vereint nach Ansicht vieler Experten das Beste aus den Welten von Mehrheits- und Verhältniswahl. ${ }^{47}$

Bei der anstehenden Reform sollte es deshalb vor allem darum gehen, das deutsche Wahlsystem in seiner bisherigen Form zu bewahren. Der Fehler eines möglichen inversen Erfolgswerts von Wählerstimmen ließe sich mit der hier vorgestellten länderweisen Sitzzuteilung beheben, ohne damit das deutsche Wahlsystem in seiner Wirkungsweise grundlegend zu verändern.

46 Vgl. den Vorschlag von Eric Linhart, a.a.O. (Fn. 17).

47 Vgl. Matthew S. Shugart / Martin P. Wattenberg, a.a.O. (Fn. 32).

\title{
Negative Stimmgewichte bei der Bundestagswahl 2009*
}

\section{Daniel Lübbert}

Aufgrund der bei der Bundestagswahl 2005 nötig gewordenen Nachwahl in Dresden ist einer breiteren Öffentlichkeit erstmals bewusst geworden, was eine kleine Gruppe von Experten schon früher bemerkt hatte: Unter bestimmten Umständen kann das geltende Wahlsystem dazu führen, dass zusätzliche Wählerstimmen einer Partei nicht nützen, sondern schaden. ${ }^{1}$ Grund dafür ist eine komplexe Wechselwirkung zwischen den Auswirkun-

* Der Autor ist Mitarbeiter der Wissenschaftlichen Dienste des Deutschen Bundestages. Der Beitrag gibt ausschließlich seine persönliche Auffassung wieder.

1 Vgl. Gerald Mann, Die unumgängliche Umkehr bei der Berechnung von Überhangmandaten: Reformvorschläge, in: ZParl, 27. Jg. (1996), H. 3, S. 398 - 404. 
gen der Erst- und Zweitstimmen im Zusammenhang mit Überhangmandaten. ${ }^{2}$ Das Bundesverfassungsgericht hat im Sommer 2008 geurteilt, dass das geltende Bundeswahlgesetz in dieser Hinsicht gegen die Grundsätze der Gleichheit und Unmittelbarkeit der Wahl verstößt und bis zum 30. Juni 2011 geändert werden muss.

Nach fast zweijähriger Diskussion in Expertenkreisen ${ }^{3}$, in Teilen der Öffentlichkeit wie im Bundestag liegen diverse Vorschläge zur Reform des Bundeswahlgesetzes vor. ${ }^{4}$ Allerdings scheint eine Entscheidung für eine der verschiedenen Lösungsmöglichkeiten weiterhin schwer zu fallen. Um hier die Orientierung zu erleichtern und mit empirischen Daten zu unterlegen, werden im Folgenden die Ergebnisse der Bundestagswahl 2009 exemplarisch untersucht, um Wirkungen und Nebenwirkungen einzelner Reformvorschläge am konkreten Einzelfall zu demonstrieren.

\section{Die Sitzverteilung im Bundestag 2009 gemäß amtlichem Endergebnis}

Die Grundlagen der Sitzverteilung im Bundestag sind die von den Parteien in den einzelnen Bundesländern errungenen Zweitstimmen und die von den Kandidaten der Parteien in den Wahlkreisen errungenen Direktmandate (vgl. Tabelle 1). Diese Zahlen sind zusammen ausreichend, um die Sitzverteilung im Bundestag zu berechnen. Diese Berechnung wurde - nach der Wahlrechtsänderung im Jahr 2008 - erstmals nach dem Verfahren von SainteLaguë / Schepers durchgeführt, nachdem bei der Wahl 2005 noch Hare / Niemeyer angewandt wurde. Aus Tabelle 2 wird ersichtlich, wie viele Sitze die Landesverbände der Parteien über ihre Liste besetzt haben. Im Vergleich wird deutlich, dass die Fraktionen der kleinen Parteien fast ausschließlich über Listenmandate besetzt werden, während die Abgeordneten der Volksparteien zu einem größeren Anteil über Direktmandate ins Parlament einziehen. Diese Tatsache ist von Bedeutung für die unten zu diskutierenden Reformvorschläge.

Tabelle 3 gibt schließlich die Gesamtzahl der Mandate im neuen Bundestag wieder. Auch bei dieser Wahl tritt der Fall auf, dass Landesverbände bereits mehr Direktmandate errungen haben, als ihnen im Ergebnis der proportionalen Zuteilung an Gesamtmandaten zustehen würde. Die überzähligen Direktmandate bleiben als Überhangmandate erhalten.

2 Siehe dazu Daniel Lübbert, Negative Stimmgewichte und die Reform des Bundestags-Wahlrechts, Info-Brief der Wissenschaftlichen Dienste des Deutschen Bundestages, Berlin 2009, www.bundestag.de/wissen/analysen/2009/negative_stimmgewichte.pdf (Abruf am 23. April 2010).

3 Vgl. Gerald Roth, Negatives Stimmengewicht und Legitimationsdefizite des Parlaments, in: NVwZ 2008, S. 1199 - 1201; Hans Meyer, Lösungsmöglichkeiten nach dem Wahlrechtsurteil des BVerfG vom 3. Juli 2008, in: DVBl. 2009, S. 137 - 146; Dieter Nohlen, Erfolgswertgleichheit als fixe Idee oder: Zurück zu Weimar? Zum Urteil des Bundesverfassungsgerichts über das Bundeswahlgesetz vom 3. Juli 2008, in: ZParl, 40. Jg. (2009), H. 1, S. 179 - 195; Joachim Behnke, Überhangmandate bei der Bundestagswahl 2009 - eine Schätzung mit Simulationen, in: ZParl, 40. Jg. (2009), H. 3, S. 620 - 636; Wilko Zicht, Stellungnahme anlässlich der Anhörung des Innenausschusses des Deutschen Bundestages am 4. Mai 2009, Berlin 2009, http://www.bundestag.de/bundestag/ausschuesse/a04/anhoerungen/Anhoerung_20/Stellungnahmen_SV/Stellungnahme_05.pdf (Abruf am 23. April 2009).

4 Vgl. Friedrich Pukelsheim, Vorschlag für $\$$ 6-7 BWahlG zur Vermeidung negativer Stimmgewichte, http://www.math.uni-augsburg.de/stochastik/pukelsheim/2008Berlin/VorschlagBWahlG.pdf (Abruf am 23. April 2010); Hans Meyer, a.a.O. (Fn. 3); Wilko Zicht, a.a.O. (Fn. 3). 


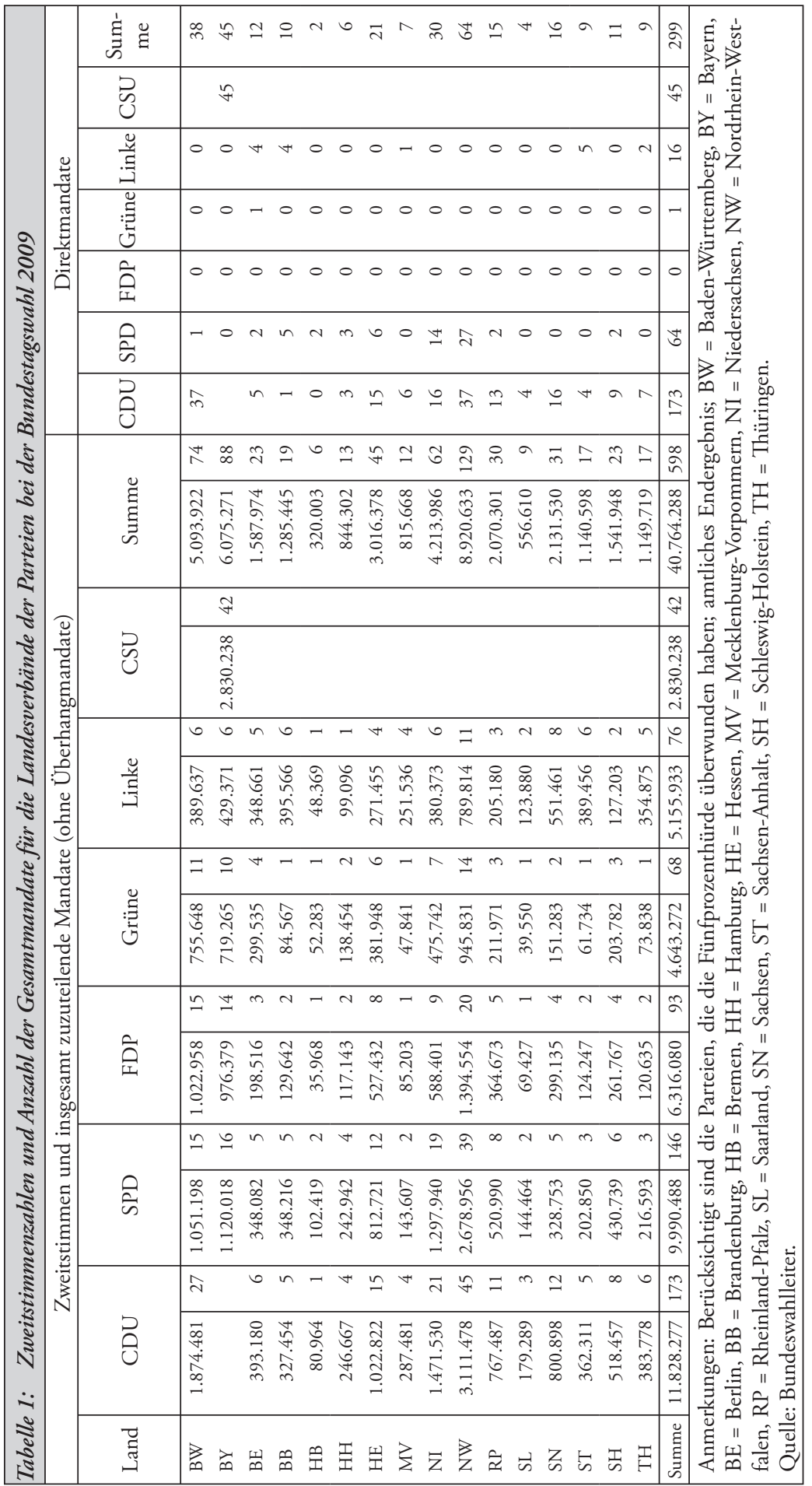




\begin{tabular}{|l|c|r|r|r|r|r|r|}
\hline \multicolumn{7}{|c|}{ Tabelle 2: Anzahl der Sitze, die ein Landesverband über die Liste füllt } \\
\hline \multicolumn{1}{|c|}{ Land } & CDU & SPD & FDP & Grüne & Linke & CSU & Summe \\
\hline BW & 0 & 14 & 15 & 11 & 6 & & 46 \\
BY & & 16 & 14 & 10 & 6 & 0 & 46 \\
BE & 1 & 3 & 3 & 3 & 1 & & 11 \\
BB & 4 & 0 & 2 & 1 & 2 & & 9 \\
HB & 1 & 0 & 1 & 1 & 1 & & 4 \\
HH & 1 & 1 & 2 & 2 & 1 & & 7 \\
HE & 0 & 6 & 8 & 6 & 4 & & 24 \\
MV & 0 & 2 & 1 & 1 & 3 & & 7 \\
NI & 5 & 5 & 9 & 7 & 6 & & 32 \\
NW & 8 & 12 & 20 & 14 & 11 & & 65 \\
RP & 0 & 6 & 5 & 3 & 3 & & 17 \\
SL & 0 & 2 & 1 & 1 & 2 & & 6 \\
SN & 0 & 5 & 4 & 2 & 8 & & 19 \\
ST & 1 & 3 & 2 & 1 & 1 & & 8 \\
SH & 0 & 4 & 4 & 3 & 2 & & 13 \\
TH & 0 & 3 & 2 & 1 & 3 & & 9 \\
\hline Summe & 21 & 82 & 93 & 67 & 60 & 0 & 323 \\
\hline Quelle: Bundeswahlleiter. &
\end{tabular}

\begin{tabular}{|c|c|c|c|c|c|c|c|}
\hline Land & $\mathrm{CDU}$ & SPD & FDP & Grüne & Linke & CSU & Summe \\
\hline BW & $37(10 \ddot{U})$ & 15 & 15 & 11 & 6 & & 84 \\
\hline BY & & 16 & 14 & 10 & 6 & 45 (3 ̈̈) & 91 \\
\hline $\mathrm{BE}$ & 6 & 5 & 3 & 4 & 5 & & 23 \\
\hline BB & 5 & 5 & 2 & 1 & 6 & & 19 \\
\hline $\mathrm{HB}$ & 1 & 2 & 1 & 1 & 1 & & 6 \\
\hline $\mathrm{HH}$ & 4 & 4 & 2 & 2 & 1 & & 13 \\
\hline $\mathrm{HE}$ & 15 & 12 & 8 & 6 & 4 & & 45 \\
\hline MV & $6(2 \ddot{U})$ & 2 & 1 & 1 & 4 & & 14 \\
\hline $\mathrm{NI}$ & 21 & 19 & 9 & 7 & 6 & & 62 \\
\hline NW & 45 & 39 & 20 & 14 & 11 & & 129 \\
\hline $\mathrm{RP}$ & $13(2 \ddot{U})$ & 8 & 5 & 3 & 3 & & 32 \\
\hline SL & 4 (1 Ü) & 2 & 1 & 1 & 2 & & 10 \\
\hline SN & 16 (4 Ü) & 5 & 4 & 2 & 8 & & 35 \\
\hline ST & 5 & 3 & 2 & 1 & 6 & & 17 \\
\hline SH & $9(1 \ddot{U})$ & 6 & 4 & 3 & 2 & & 24 \\
\hline $\mathrm{TH}$ & $7(1 \ddot{U})$ & 3 & 2 & 1 & 5 & & 18 \\
\hline Summe & $194(21 \ddot{U})$ & 146 & 93 & 68 & 76 & 45 (3 Ü) & $622(24 \ddot{U})$ \\
\hline \multicolumn{8}{|c|}{$\begin{array}{l}\text { Anmerkung: Die Schreibweise „37 (10 Ü)“ bedeutet: } 37 \text { Mandate insgesamt, darunter zehn Überhang- } \\
\text { mandate. } \\
\text { Quelle: Bundeswahlleiter. }\end{array}$} \\
\hline
\end{tabular}


Der Bundestag wächst so von seiner regulären Sitzzahl gemäß Bundeswahlgesetz (598 Abgeordnete) um weitere 24 Mandate auf insgesamt 622 Abgeordnete an. Die Zahl der Überhangmandate liegt damit um 50 Prozent über dem bisherigen historischen Höchststand (16 im Jahr 2005). Ungewohnt ist auch, dass die Überhangmandate ausschließlich den beiden Unionsparteien zugutekommen. Beide Effekte wurden allerdings bereits vor der Wahl vorausgesagt. ${ }^{5}$ Allein der CDU-Landesverband Baden-Württemberg erringt zehn Überhangmandate. Mit der CSU, die in Bayern drei Überhangmandate erreicht, tritt zum ersten Mal in der Geschichte der Bundesrepublik der Fall ein, dass eine Partei sogar auf Bundesebene mehr Direktmandate bekommt, als ihr im Ergebnis der Oberzuteilung nach Zweitstimmen zustehen würde. Diese „externen Überhangmandate“ sind bei der anstehenden Reform des Wahlrechts von besonderer Bedeutung. Die CSU ist damit allerdings nicht so allein, wie es zunächst erscheinen mag: Die CDU hat bundesweit 173 Direktmandate per Erststimmen gewonnen - exakt so viele, wie ihr laut proportionaler Zuteilung nach Zweitstimmen an Mandaten insgesamt zustanden. Mit nur einem Direktmandat mehr (oder alternativ mit weniger Zweitstimmen) hätte sie somit ebenfalls nicht nur interne, sondern sogar externe Überhangmandate erzielt.

Teilt man die Stimmenzahlen durch die Mandatszahlen, so ergibt sich als Verhältnis das Vertretungsgewicht der Mandate. Es verdeutlicht, wie viele Wähler im Mittel mit ihrer Zweitstimme hinter einem Abgeordneten des jeweiligen Landesverbandes stehen. Der bundesweite Mittelwert liegt für die Wahl 2009 bei circa 68.000 Stimmen pro Bundestagssitz (ohne Überhangmandate) - und damit aufgrund der geringeren Wahlbeteiligung deutlich niedriger als im Jahr 2005 (circa 76.000). Dass die tatsächlichen Werte teils erheblich von diesem Mittel abweichen, liegt - neben Rundungseffekten in kleinen Ländern - primär daran, dass die Vertretungsgewichte durch Überhangmandate systematisch verändert werden. Besonders deutlich wird dies bei der CDU Baden-Württemberg, die bei 37 Direktmandaten zehn Überhangmandate erhält. Dadurch verringert sich das Verhältnis Zweitstimmen pro Sitz auf circa 50.600. Im Mittel über alle Bundesländer weichen deshalb auch die mittleren Vertretungsgewichte - beziehungsweise deren Kehrwerte, die Erfolgswerte der Wählerstimmen - der verschiedenen Parteien deutlich voneinander ab: Zweitstimmen für die SPD erzielen bundesweit 4,2 Prozent weniger Sitze als die durchschnittliche Wählerstimme, während CDU-Zweitstimmen zu einem um 7,5 Prozent über dem Durchschnitt liegenden Sitzerfolg führen. Diese Unterschiede sind zum ganz überwiegenden Teil auf den Effekt der Überhangmandate zurückzuführen.

\section{Analyse negativer Stimmgewichte}

Um zu untersuchen, welche Änderungen sich an den Sitzzahlen ergeben hätten, wenn die Stimmenzahlen leicht unterschiedlich ausgefallen wären, sollen im Folgenden Änderungen einer genau definierten Art betrachtet werden: Für jeden einzelnen Landesverband der sechs im Bundestag vertretenen Parteien wird berechnet, wie viele Wählerstimmen mehr oder weniger erforderlich gewesen wären, um die nächstliegende Änderung der Sitzverteilung auszulösen. Nicht betrachtet werden hingegen weitergehende Sitzverschiebungen bei noch größeren Stimmenunterschieden, ebenso wenig wie gleichzeitige Veränderungen bei 
mehreren Landesverbänden, Veränderungen bei den Erststimmen oder bei der Wahlbeteiligung.

Die eintretenden Veränderungen können unterschiedlicher Art sein. Wenig erstaunlich ist es beispielsweise, dass ein Landesverband aufgrund erhöhter Stimmenzahl einen zusätzlichen Sitz erhält - sei es zu Lasten einer anderen Partei oder eines anderen Landesverbandes der eigenen Partei. Es treten jedoch auch überraschendere Fälle auf, die in Tabelle 4 zusammengefasst sind.

\begin{tabular}{|c|c|c|}
\hline Hätte die ... & $\begin{array}{c}\ldots \text { Zweitstimmen } \\
\text { bekommen ... }\end{array}$ & ... dann hätte die ... \\
\hline CDU Baden-Württemberg & 60.735 weniger & CDU-Niedersachsen 1 Sitz mehr. \\
\hline CDU Hessen & 30.395 weniger & CDU-Niedersachsen 1 Sitz mehr. \\
\hline CDU Mecklenburg-Vorpommern & 47.930 weniger & CDU-Niedersachsen 1 Sitz mehr. \\
\hline CDU Rheinland-Pfalz & 48.833 weniger & CDU-Niedersachsen 1 Sitz mehr. \\
\hline CDU Saarland & 8.181 weniger & CDU-Niedersachsen 1 Sitz mehr. \\
\hline CDU Sachsen & 13.801 weniger & CDU-Niedersachsen 1 Sitz mehr. \\
\hline CDU Schleswig-Holstein & 5.133 weniger & CDU-Niedersachsen 1 Sitz mehr. \\
\hline CDU Thüringen & 7.341 weniger & CDU-Niedersachsen 1 Sitz mehr. \\
\hline SPD Brandenburg & 42.757 weniger & SPD-Bayern 1 Sitz mehr. \\
\hline SPD Bremen & 600 weniger & SPD-Bayern 1 Sitz mehr. \\
\hline \multicolumn{3}{|c|}{$\begin{array}{l}\text { Anmerkung: Aufgeführt ist jeweils nur die erste Sitzveränderung bei kleinen Stimmenunterschieden } \\
\text { Hinzu kommen weitere Fälle, in denen aufgrund noch höherer Stimmeneinbußen ein zweiter oder wei } \\
\text { tere Sitze verschoben werden („negatives Stimmgewicht zweiter beziehungsweise höherer Ordnung“). } \\
\text { Quelle: Eigene Berechnungen. }\end{array}$} \\
\hline
\end{tabular}

Diese konzentriert sich auf diejenigen Fälle, in denen die Veränderung der Sitzzahl den Erwartungen zuwider läuft. Es handelt sich bei all diesen um das Phänomen des negativen Stimmgewichts. Am Beispiel des letzten Eintrags in Tabelle 4 kann es wie folgt erklärt werden (derselbe Mechanismus gilt für alle in der Tabelle genannten Fälle): Hätte die SPD in Bremen 600 Stimmen weniger bekommen, so hätte dieser geringe Verlust auf der Ebene der Oberzuteilung auf Bundesebene noch zu keinem Sitzverlust für die SPD geführt. Bei der Unterzuteilung an die einzelnen Landesverbände der SPD hingegen ergibt sich eine Verschiebung: Zuvor hatte die SPD Bremen knapp einen zweiten Sitz errungen, während die SPD Bayern ebenso knapp am Gewinn eines weiteren Listenmandats gescheitert war. Durch den rechnerischen Abzug von genau 600 Stimmen in Bremen kehrt sich dieses Verhältnis um, so dass nun die SPD Bayern einen zusätzlichen Sitz zugeteilt bekommt. Die SPD Bremen muss dafür theoretisch ein Listenmandat abgeben, was für sie jedoch zu keinem praktischen Verlust führt, da sie über Erststimmen auch zwei Direktmandate errungen hat. Beide bleiben erhalten; der Unterschied besteht darin, dass ein Direktmandat in ein Überhangmandat umgewandelt wird.

Im Endeffekt würde sich der Bundestag so um einen Sitz vergrößern, den ein zusätzlicher SPD-Abgeordneter aus Bayern einnehmen könnte - allein deshalb, weil seine eigene Partei Stimmen verloren hätte. In der Realität hingegen hat die SPD Bremen die fraglichen 600 Zweitstimmen doch erhalten. Diese haben so umgekehrt zu einem rechnerischen Sitzverlust derjenigen Partei geführt, der sie eigentlich nutzen sollten - den Stimmen dieser Wähler kommt ein „negatives Gewicht“ zu. 
Bei fortgesetzter Berechnung der Folgen hypothetischer Zweitstimmen-Verluste ergibt sich, dass ab einer Schwelle von -68.509 Zweitstimmen die SPD Bremen auch ihren zweiten Listenplatz verlöre, diesmal an die SPD Nordrhein-Westfalen. Wiederum erlitte sie selbst dadurch keinen Sitzverlust; vielmehr würde auch ihr zweites Direktmandat in ein weiteres Überhangmandat umgewandelt. Die SPD-Fraktion im Bundestag hätte sich also aufgrund eines solchen Stimmenverlustes um einen zweiten Sitz vergrößert. Vom negativen Gewicht ihrer Zweitstimmen sind demnach keineswegs nur wenige hundert, sondern rund 70.000 der insgesamt circa 102.000 SPD-Wähler in Bremen betroffen.

Ein ähnlicher Fall tritt in Brandenburg auf, wo ein Verlust von 42.757 Stimmen (oder mehr) für den Landesverband der SPD ebenfalls zu einem weiteren SPD-Sitz in Bayern geführt hätte, ohne dass die SPD-Brandenburg selbst einen Sitz verloren hätte. Auf Seiten der CDU zeigt sich dasselbe Phänomen: Büßte einer der in Tabelle 4 dargestellten Landesverbände der CDU die dort genannte Anzahl (oder mehr) an Zweitstimmen ein, so würde über einen ähnlichen Mechanismus jeweils die CDU Niedersachsen, die zuvor knapp vor einem zusätzlichen Mandat stand, einen Sitz hinzugewinnen. Der ,abgebende“ CDU-Landesverband hingegen würde selbst keinen Sitzverlust erleiden, sondern lediglich ein Direktmandat in ein weiteres Überhangmandat umwandeln.

Insgesamt sind gemäß Tabelle 4 zwei Parteien mit zusammen zehn Landesverbänden vom negativen Stimmgewicht betroffen. Es handelt sich dabei um die sieben Landesverbände der CDU, die tatsächlich Überhangmandate erzielt haben, sowie um drei weitere (CDU Hessen; SPD Brandenburg und SPD Bremen), die zwar kein eigenes Überhangmandat erringen konnten, aber zumindest knapp davor standen. Diese zehn „ÜberhangKandidaten" sind bereits aus Tabelle 2 einheitlich daran erkennbar, dass keiner dieser Landesverbände Listenmandate zugeteilt bekommen hat. Die CSU hingegen, die als eigenständige Partei zur Wahl antritt, ist ein besonderer Fall: Auch sie erringt zwar erstmals drei (externe) Überhangmandate. Diese führen aber nicht zu negativem Stimmgewicht, weil außerhalb von Bayern kein zweiter CSU-Landesverband existiert, der an einer Sitzverschiebung teilhaben könnte. Die drei kleineren Parteien schließlich sind am Phänomen des negativen Stimmgewichts auf den ersten Blick gar nicht beteiligt, weil sie in der Regel keine Direktmandate erringen - wenn aber doch, so bleiben sie zumindest von Überhangmandaten weit entfernt.

Zumindest für die Linke gilt dies bei genauerer Betrachtung nur eingeschränkt: Die Partei hat in Sachsen-Anhalt fünf Direktmandate sowie zusätzlich ein Listenmandat erworben. Unterstellt man hier kleine bis mittlere Stimmenverluste des Landesverbandes, so ginge zunächst das eine Listenmandat verloren (an die SPD Bayern), ab -76.935 Zweitstimmen in Sachsen-Anhalt träte außerdem die Linke Niedersachsen einen Sitz an die CDU ab. Bei weitergehenden Verlusten jedoch verlöre die Linke Sachsen-Anhalt kein weiteres Mandat, sondern ihre Direktmandate würden zu Überhangmandaten. Im Gegenzug erhielte (ab -78.245 Stimmen in Sachsen-Anhalt) die Linke in Niedersachsen sowie (ab -149.080 Stimmen) in Nordrhein-Westfalen je ein Listenmandat neu hinzu. Auch die Linke kann insofern als vom negativen Stimmgewicht indirekt betroffene Partei gelten.

In der Gesamtschau bestätigt sich also, was von Beobachtern zuvor erwartet worden war: Negative Stimmgewichte sind in den Ergebnissen der Bundestagswahl 2009 vielfach zu beobachten. Alle Fälle ihres Auftretens sind mit Überhangmandaten verbunden. Diese müssen vom jeweiligen Landesverband nicht notwendigerweise bereits errungen worden sein. Es reicht, wenn sie in greifbare Nähe gerückt sind; schon dadurch können negative 
Stimmgewichte entstehen. Umgekehrt gilt, dass alle Fälle von greifbar nahen oder von tatsächlich aufgetretenen (internen) Überhangmandaten unvermeidlich auch zu negativen Stimmgewichten geführt haben. Betroffen vom negativen Gewicht ihrer Stimme sind gemäß Tabelle 4 mindestens einige hunderttausend, tatsächlich aber noch deutlich mehr Wähler von CDU und SPD - sowie indirekt der Linken - in ganz Deutschland. Diese Wähler hätten ihrer präferierten Partei dadurch nutzen können, dass sie der Wahl fern geblieben wären.

\section{Einzelschicksale}

Tabelle 4 verdeutlicht, dass vom negativen Gewicht ihrer Stimmen die Wähler von insgesamt zehn Landesverbänden der großen Parteien betroffen sind. Die wegen zu vieler Stimmen entgangenen Bundestagssitze konzentrieren sich jedoch auf nur zwei Landesverbände: die CDU Niedersachsen und die SPD Bayern. In beiden Fällen lassen sich die Leidtragenden persönlich identifizieren: Es handelt sich um zwei bisherige Abgeordnete, die jeweils bereits seit mehreren Wahlperioden im Bundestag saßen und den Wiedereinzug nun deswegen verpasst haben, weil ihre Parteien in anderen Bundesländern „zu viele“ Zweitstimmen erhalten haben. In Niedersachsen stand Jochen-Konrad Fromme auf Platz 11 der CDULandesliste. Hätte etwa die CDU Saarland 8.181 oder die CDU Schleswig-Holstein 5.133 Zweistimmen weniger bekommen, so hätte er zusätzlich in den Bundestag einziehen können, ohne dass ein anderer Abgeordneter dafür hätte weichen müssen.

Noch prägnanter ist der Fall des Abgeordneten Axel Berg ${ }^{6}$, der auf Platz 17 der SPDLandesliste Bayern stand. Er ist von einem außerordentlich knappen Fall von negativem Stimmgewicht betroffen: Hätte die SPD Bremen 600 Stimmen weniger erhalten, so wäre Berg zusätzlich ins Parlament gekommen, wiederum ohne dass ein anderes Mitglied des Bundestages dafür hätte weichen müssen. Ebenfalls in den Bundestag eingezogen wäre er, wenn die SPD Bremen nicht weniger, sondern 594 Zweitstimmen mehr bekommen hätte. Zwischen -600 und +594 Zweitstimmen in Bremen lag für die SPD ein sehr schmales Fenster, innerhalb dessen sie einen Abgeordneten einbüßte. Genau in dieses enge Intervall ist das tatsächliche Wahlergebnis gefallen.

\section{Effekte einiger Reformvorschläge für das Bundeswablgesetz}

Wenn Wählerstimmen für eine Partei dieser schaden können, so ist dies - wie vom Bundesverfassungsgericht festgestellt - schwerlich mit den Grundsätzen der Gleichheit und der

6 In diesem Fall kommen weitere „Knappheiten“ hinzu: Als Direktkandidat in seinem Wahlkreis ist Axel Berg um circa 1.500 Stimmen am Gewinn eines Direktmandats gescheitert; als Listenkandidat fehlten 594 Zweitstimmen für seinen Landesverband, um seinen Wiedereinzug in den Bundestag zu ermöglichen. Daneben ist er einer der sehr wenigen Leidtragenden der Umstellung des Zuteilungsverfahrens auf Sainte-Laguë / Schepers (das ansonsten deutliche Vorteile mit sich bringt, weil es unlogische Sprünge und mathematische „Inkonsistenzen“ bei der Zuteilung vermeidet): Nach dem alten Verfahren von Hare / Niemeyer wäre Berg selbst bei unveränderter Stimmenzahl über einen zusätzlichen Sitz in den Bundestag eingezogen. 
Unmittelbarkeit der Wahl vereinbar. Die oben geschilderten Tatsachen zeigen daher deutlich, dass das Bundeswahlgesetz der Korrektur bedarf. ${ }^{7}$

Um das komplexe Feld der Lösungsmöglichkeiten auszuleuchten, sollen im Folgenden die Ergebnisse der Bundestagswahl 2009 verwendet werden, um die Wirkungen und Nebenwirkungen der wichtigsten Reformvorschläge im Einzelfall zu analysieren. Dem Vorgehen liegt die vereinfachende Annahme zugrunde, dass das Stimmverhalten der Wähler sowohl für die Erst- als auch für die Zweitstimme - unter einem veränderten Wahlsystem unverändert bliebe.

Eine der einfachsten Möglichkeiten zur Verhinderung des negativen Stimmgewichts läge darin, das Wahlsystem auf eine reine Verhältniswahl umzustellen (das heißt Erststimmen und Direktmandate abzuschaffen). Dies könnte Nachteile für die Verankerung von Politikern in den Wahlkreisen vor Ort nach sich ziehen, würde aber zumindest das Entstehen von Überhangmandaten und von negativen Stimmgewichten unmöglich machen. Die Auswirkungen auf die Fraktionsstärken im Bundestag wären begrenzt, wie aus Tabelle 5 ersichtlich ist: Die in Tabelle 3 in Klammern genannten Überhangmandate würden entfallen; die vor der Klammer genannten Stärken der jeweiligen Landesverbände würden um die entsprechende Zahl reduziert.

Eine ebenso einfache Alternative bestünde darin, ein reines Mehrheitswahlrecht einzuführen (Abschaffung der Zweitstimme und der Listenmandate). Der Bundestag würde dann allein über Direktmandate besetzt. Die Auswirkungen auf die Fraktionsstärken könnten grob abgeschätzt werden, indem man die in Tabelle 1 wiedergegebenen Direktmandatszahlen verdoppelt. Die Auswirkungen auf Optionen der Regierungsbildung und auf die Wahlchancen kleinerer Parteien wären sehr bedeutend. Um eine solche Reform jedoch zunächst rein technisch umzusetzen, müsste zuvor die Zahl der Wahlkreise verdoppelt und ihre jeweiligen Grenzen neu gezogen werden. Dabei käme es im Sinne der Chancengleichheit aller Wählerstimmen entscheidend darauf an, dass jeder dieser Wahlkreise annähernd gleich viele Wähler umfasst; gegenwärtig weichen die Wahlkreisgrößen um bis zu +/-20 Prozent nach oben und unten $\mathrm{ab}$. Eine solche vollständige Neudefinition aller Wahlkreise stellt eine mathematisch wie politisch schwierige Operation dar. Daher kann diese Option hier nicht weiter analysiert werden; sie fehlt deshalb auch in Tabelle 5.

Als Mittelweg zwischen Mehrheits- und Verhältniswahl wird auch ein sogenanntes Grabenwahlsystem vertreten: Dabei würde wie bisher die erste Hälfte der Sitze über Direktmandate aus den bestehenden 299 Wahlkreisen vergeben. Diese würden im zweiten Schritt allerdings nicht mehr mit den Listenmandaten verrechnet. Vielmehr würde sich die proportionale Berechnung nach Zweitstimmen, anders als bisher, nur noch auf die zweite Hälfte der Mandate auswirken. Die Konsequenzen für die Fraktionsstärken werden aus Tabelle 5 deutlich: Profitiert hätten die Unions-Parteien, während alle anderen Mandate eingebüßt hätten. Besonders stark gelitten hätten die Grünen und die FDP, die nur eines

7 Dies gilt unabhängig von der im Frühjahr 2008 im Bundeswahlgesetz vollzogenen Umstellung des Sitzzuteilungsverfahrens von Hare / Niemeyer auf Sainte-Laguë / Schepers: Nach den obigen Feststellungen ist offensichtlich, dass negative Stimmgewichte - ebenso wie bei der nach Hare I Niemeyer ausgewerteten Wahl 2005 - auch mit dem neuen Verfahren auftreten können. Umgekehrt hätten die Stimmenergebnisse von 2009, ausgewertet nach dem alten Zuteilungsverfahren, ebenfalls zahlreiche Fälle von negativen Stimmgewichten gezeitigt, wenn auch die Details etwas anders als in Tabelle 4 ausgefallen wären. 
beziehungsweise gar kein Direktmandat errungen haben. Angesichts dieser Verschiebungen hätte auch die Regierungsbildung anders ablaufen können: Eine Unions-Alleinregierung aus CDU und CSU hätte bereits ohne die FDP eine Mehrheit von 27 Stimmen gehabt.

\begin{tabular}{|c|c|c|c|c|c|c|c|}
\hline \multicolumn{8}{|c|}{$\begin{array}{l}\text { Tabelle 5: Reformvorschläge zum deutschen Wahlsystem zwecks Abschaffung des negativen } \\
\text { Stimmgewichts und ihre Auswirkungen auf die Fraktionsstärken im 17. Deutschen } \\
\text { Bundestag }\end{array}$} \\
\hline Wahlsystem-Alternative & $\mathrm{CDU}$ & SPD & FDP & Grüne & Linke & $\mathrm{CSU}$ & Summe \\
\hline $\begin{array}{l}\text { Status quo (Bundestagswahl vom } \\
\text { 27. September 2009, ausgewertet } \\
\text { nach geltendem BWahlG) }\end{array}$ & 194 & 146 & 93 & 68 & 76 & 45 & 622 \\
\hline Reine Verhältniswahl & 173 & 146 & 93 & 68 & 76 & 42 & 598 \\
\hline Grabenwahlsystem & 260 & 137 & 46 & 35 & 54 & 66 & 598 \\
\hline Wahl getrennt nach Ländern & 195 & 148 & 91 & 69 & 76 & 45 & 624 \\
\hline Bundesweite Ausgleichsmandate & 233 & 196 & 124 & 91 & 101 & 56 & 801 \\
\hline $\begin{array}{l}\text { Kompensation aus anderen } \\
\text { Landeslisten }\end{array}$ & 173 & 146 & 93 & 68 & 76 & 45 & 601 \\
\hline $\begin{array}{l}\text { Ebenso, plus Ausgleichsmandate } \\
\text { nur in Bayern }\end{array}$ & 173 & 148 & 94 & 69 & 77 & 45 & 606 \\
\hline \multicolumn{8}{|c|}{$\begin{array}{l}\text { Anmerkung: Die Auswirkungen auf Optionen der Regierungsbildung sind geringer als bei den reinen } \\
\text { Sitzzahlen: In jedem dieser Szenarien hätte die schwarz-gelbe Koalition weiterhin eine Mehrheit. Einzig } \\
\text { im Falle des Grabenwahlsystems würde die FDP für die Regierungsbildung entbehrlich, weil CDU/CSU } \\
\text { zusammen eine eigenständige Kanzlermehrheit aufbrächten. } \\
\text { Quelle: Eigene Berechnungen. }\end{array}$} \\
\hline
\end{tabular}

Eine andere Reformmöglichkeit greift bei der föderalen Struktur an: Bisher werden zunächst die Stimmen jeder Partei auf Bundesebene addiert, die Fraktionsstärken im Bundestag daraus berechnet und anschließend Sitze jeder Partei auf ihre jeweiligen Landesverbände verteilt. Eine Alternative wäre, die Verrechnung auf Bundesebene abzuschaffen und die Wahl nach Bundesländern getrennt auszuwerten, die den Bundestag dann jeweils separat mit Abgeordneten der verschiedenen Parteien beschicken. Generell ist es möglich, die zahlenmäßigen Auswirkungen einer solchen Lösung zu berechnen. Dabei wäre allerdings vorab zu entscheiden, ob die Fünfprozenthürde weiterhin auf Bundesebene oder aber individuell in den Ländern angewandt werden soll. Auch müsste geklärt werden, ob die Gesamtzahl der Abgeordneten pro Bundesland gemäß der Zahl der Einwohner beziehungsweise Wahlberechtigten vorab festgelegt werden soll, oder ob sie weiterhin (wie im bisherigen System) erst nach der Wahl feststehen und dabei auch in Abhängigkeit von der regionalen Wahlbeteiligung in den einzelnen Ländern schwanken darf. Es sind so verschiedene Szenarien denkbar; eines davon ist in Tabelle 5 exemplarisch wiedergegeben. Das Ergebnis zeigt, dass die Fraktionsstärken sich in diesem Szenario nur sehr geringfügig ändern würden. Negative Stimmgewichte würden so sicher verhindert; gegen eine solche Lösung sprechen allerdings staatstheoretische Gründe (insbesondere der „unitarische Charakter“ des Organs Bundestags).

Eine weitere Reformvariante besteht darin, das System aus Erst- und Zweitstimmen unverändert zu lassen und auch Überhangmandate weiterhin zu erlauben, diese aber durch 
zusätzliche Ausgleichsmandate für die anderen Parteien politisch zu „neutralisieren“. Im Detail existieren verschiedene Möglichkeiten zur Umsetzung dieses Grundgedankens. Eine noch vergleichsweise „sparsame“ Variante besagt, dass die Gesamtgröße des Parlaments so lange um jeweils ein Mandat erhöht und die Sitzzuteilung wiederholt neu berechnet wird, bis gerade das letzte Überhangmandat in ein reguläres Mandat umgewandelt ist. ${ }^{9}$ Selbst diese Lösung, die die Parlamentsvergrößerung durch Ausgleichsmandate auf das erforderliche Minimum begrenzt, führt im Fall der Wahl 2009 auf einen Bundestag mit 801 Sitzen (vgl. Tabelle 5). Diese Variante dürfte damit schon aus Kostengründen ausfallen.

Eine letzte Lösungsmöglichkeit besagt, dass (interne) Überhangmandate einzelner Landesverbände weiterhin zugelassen wären, diese aber über andere Landeslisten derselben Partei kompensiert würden, die dann entsprechend weniger Listenmandate zugeteilt bekämen. Im Falle der Wahl 2009 wäre davon allein die CDU betroffen, weil außerhalb der Union keine Partei Überhangmandate errungen hat und weil die CSU über keinen zweiten Landesverband verfügt, der zur Kompensation herangezogen werden könnte. Auch innerhalb der CDU ist die Auswahl der Landesverbände, die kompensieren könnten, eng begrenzt: Sieben davon haben selbst Überhangmandate errungen, ein achter (Hessen) stand kurz davor. Prinzipiell verfügen daher nur die verbleibenden sieben CDU-Landesverbände überhaupt über Listenmandate, deren Zahl theoretisch reduziert werden könnte. Zur Verteilung auf diese sind zahlreiche Vorschläge gemacht worden. ${ }^{10}$ Hier soll der Variante von Pukelsheim gefolgt werden: Demnach bekämen vier Landesverbände der CDU (die drei Stadtstaaten sowie Sachsen-Anhalt) jeweils eines, Brandenburg vier, Niedersachsen fünf und NordrheinWestfalen acht Listenmandate gegenüber dem Status quo weniger zugeteilt. ${ }^{11}$ Eine andere Möglichkeit existiert im konkreten Fall der Wahl 2009 prinzipiell nicht, weil die CDU auf Bundesebene insgesamt nur 21 Listenmandate zugeteilt bekommen hat - exakt gleich viele, wie Überhangmandate zu kompensieren sind. Unvermeidlich muss daher jedes einzelne dieser Listenmandate zur Kompensation herangezogen werden.

Die resultierenden Sitzzahlen (vgl. Tabelle 5) sind identisch mit dem Fall der reinen Verhältniswahl, soweit die reinen Fraktionsstärken betrachtet werden, unterscheiden sich aber im Hinblick auf deren regionale Zusammensetzung. Einzige Ausnahme von der Regel ist die CSU, die ihre drei (externen) Überhangmandate behalten darf, weil sie nicht kompensiert werden können; die Gesamtgröße des Parlaments erhöht sich so von 598 auf 601. Diese Tatsache ist vielfach als „CSU-Problem“ bezeichnet worden. Anzumerken ist hier, dass es sich dabei nicht um ein verfassungsrechtliches oder mathematisches Problem handelt: Mangels eines zweiten Landesverbands der CSU können ihre Überhangmandate nicht zu negativen Stimmgewichten führen. Sie könnten daher theoretisch - folgt man der Rechtsprechung des Bundesverfassungsgerichts - erhalten bleiben. Dies würde allerdings wohl zu Unverständnis bei anderen Parteien führen, deren Überhangmandate zuvor kompensiert wurden und die nun eine Ungleichbehandlung vermuten könnten. Insofern liegt tatsächlich ein (politisches) Problem vor. Um dieses zu lösen, kann wiederum die Vergabe

9 Vgl. Friedrich Pukelsheim / Sebastian Maier, Parlamentsvergrößerung als Problemlösung für Überhangmandate, Pattsituationen und Mehrheitsklauseln, in: ZParl, 39. Jg. (2008), H. 2, S. 312 322.

10 Vgl. Gerald Mann, a.a.O. (Fn. 1); Joachim Behnke, Das Wahlsystem der Bundesrepublik Deutschland: Logik, Technik und Praxis der Verhältniswahl, Baden-Baden 2007.

11 Vgl. Friedrich Pukelsheim, a.a.O. (Fn. 4). 
von Ausgleichsmandaten erwogen werden. Diese bliebe nun strikt auf das betroffene Land Bayern beziehungsweise auf die verbleibenden externen Überhangmandate beschränkt. In Bayern würden die drei Überhangmandate der CSU durch zwei zusätzliche Sitze für die SPD sowie je einen für FDP, Bündnis 90/Die Grünen und Die Linke ausgeglichen. Der Bundestag wüchse so auf 606 Sitze geringfügig weiter an (vgl. Tabelle 5).

Eine ähnliche Lösung bietet sich an, falls die CDU ein oder mehrere weitere Direktmandate gewonnen hätte und so ebenfalls in den Bereich externer Überhangmandate gekommen wäre, die nicht mehr über ihre Landesverbände kompensiert werden könnten. In einem solchen Fall könnten die anderen Parteien einige Ausgleichsmandate auf Bundesebene zugesprochen bekommen, die über den regulären Mechanismus der Unterzuteilung auf ihre Landesverbände verteilt würden.

\section{5. „Minimal-invasive“ Lösung: Kompensation des Überhangs}

Die Bundestagswahl 2009 hat in mehrfacher Hinsicht neuartige Ergebnisse bei der Sitzzuteilung mit sich gebracht: Die Zahl der Überhangmandate liegt höher als je zuvor. Mit der CSU hat erstmals eine Partei externe Überhangmandate errungen; eine zweite, die CDU, stand sehr kurz davor. Auch der Effekt des negativen Stimmgewichts ist wiederum vielfach zutage getreten. Eine Besonderheit seines jüngsten Auftretens liegt darin, dass ein Ausgang besonders knapp war: Für die SPD ging es in Bremen um genau 600 Stimmen, die sie hätte verlieren müssen, um einen Sitz hinzuzugewinnen.

Im Vorfeld der Wahl ist vielfach gemutmaßt worden, dass die Volksparteien eine Korrektur des Wahlsystems schon vor September 2009 vor allem deshalb nicht mitgetragen hätten, weil sie von den zu erwartenden Überhangmandaten noch profitieren wollten. Ironischerweise sind es hunderttausende Wähler gerade dieser Volksparteien, die nun gemäß dem Wahlergebnis von einem negativen Gewicht ihrer eigenen Stimme betroffen sind.

Alle Varianten, das Bundeswahlgesetz - wie vom Bundesverfassungsgericht gefordert zu korrigieren, sind durch gewisse Nebenwirkungen gekennzeichnet. So würde die Einführung eines reinen Mehrheitswahlsystems das Parteienspektrum der Bundesrepublik vermutlich stark verändern. Auch ein Grabenwahlsystem als vermeintlich „schonendere“ Lösung würde voraussichtlich die kleineren Parteien stark dezimieren, hätte 2009 die FDP bei der Regierungsbildung entbehrlich gemacht und eine CDU/CSU-Alleinregierung ermöglicht. Weiterhin scheiden Ausgleichsmandate auf Bundesebene wegen übermäßiger Parlamentsvergrößerung um fast 200 Sitze aus. Allenfalls könnte man die reguläre Sitzzahl vorab deutlich reduzieren, um sie danach im Einzelfall durch Ausgleichsmandate wieder ansteigen zu lassen.

Vor diesem Hintergrund erscheint schließlich der Vorschlag einer Kompensation von Überhangmandaten über andere Landeslisten derselben Partei als „minimal-invasive“ Lösung mit den bisher vermutlich geringsten Nebenwirkungen. Zweifellos bringt auch diese Variante unerwünschte Effekte mit sich. Insbesondere wird hier die Verbesserung des Parteien-Proporzes mit einer Einbuße beim Länder-Proporz erkauft. Dennoch erscheint diese Lösung bisher am besten geeignet, das Gewicht jeder einzelnen Wählerstimme unter allen Umständen wieder ins Positive zu wenden. 